\title{
artículos
}

\section{Proyectos para la Plaza de España de Melilla Comandancia General versus Ayuntamiento}

\author{
Rosario Camacho Martínez \\ Universidad de Málaga
}

PALABRAS CLAVE: Arquitectura s. XX/ Urbanismo/ Melilla

\section{RESUMEN}

Se analizan los proyectos realizados para el solar más significativo de la Plaza de España de Melilla, en la década de 1920. El primero es una evocación regionalista para un edificio militar. Los otros cuatro son proyectos para el Aynntamiento y, con una excepción, teniendo como base el neoárabe, ofrecen mayor modernidad acusando el impacto del art déco, que se manifiesta plenamente en el edificio construido por el arquitecto municipal Enrique Nieto.

\section{ABSTRACT}

This article is devoted to the analysis of several projects for the most important plot of the main square of Melilla, about 1920. The first is a regionalist evocation for a military building. The other four are projects for the town-hall that, except one, are the newarab style as model, and offer more modernité with the art déco impact, that is more evident in the building of the architect Enrique Nieto.

\section{INTRODUCCIÓN.}

La ciudad de Melilla, situada a orillas del Mediterráneo, en el N. de África, y asentamiento de diferentes culturas, entra en la historia de España a partir de su ocupación, en 1497, por las naves del Duque de Medina Sidonia mandadas por Pedro de Estopiñán, quien desembarcó en una población en ruinas, la antigua Rusaddir. Para su defensa se construyó una cerca de murallas, origen de la ciudad-fortaleza, concebida como guarnición militar y presidio, que condicionó su morfología de urbe cerrada, durante cuatro siglos, en los cuales no hubo una especial atención de la Corona hacia las plazas del norte de África.

* CAMACHO MARTíNEZ, R.: "Proyectos para la Plaza de España de Melilla: Comandancia General versus Ayuntamiento", Boletín de Arte, n²8, Departamento de Historia del Arte, Universidad de Málaga, 2007, págs 227-240 
Pero desde mediados del siglo XIX los acontecimientos políticos sientan las bases para el desarrollo de la moderna Melilla. Inglaterra y Francia, habian iniciado una carrera de expansión y colonización en Asia y Africa, que provocó una mayor atención de España hacia Marruecos, especialmente, a sus plazas del norte de África. Tras la Guerra de Tetuán (1859), el Tratado de Paz de 1860 señala las nuevas funciones que va a desempeñar Melilla y propiciaron su transformación, siendo la fecha de 1862 la que marca el inicio de la expansión de la ciudad, señalándose los límites del nuevo territorio mediante el alcance de un disparo de cañón realizado desde uno de los fuertes de la plaza, cumpliendo así lo designado en el Tratado.

Desde 1904 Melilla contaba con un gobierno militar independiente de la Capitanía General de Granada, en 1910 la Comandancia General de Melilla se transforma en Capitanía General, y la formalización del Protectorado Español sobre Marruecos, se produjo en 1912, determinando sensibles cambios en la estructura militar de la ciudad. Hay que citar dos organismos que tienen una importancia capital en la construcción de la ciudad: la Comandancia de Obras (1893), dependiente de la Comandancia General de Melilla, que constituyó la "cantera" de la que surgieron los ingenieros militares que participaron activamente en la urbanización de Melilla, y la Junta de Arbitrios, dependiente del Ministerio de la Guerra (1883), que gobernaba Melilla y fue el antecedente del Ayuntamiento1. A partir de 1927 la Junta de Arbitrios, la institución que más arquitectura financió en Melilla, se transformó en Municipal y en 1930 en Ayuntamiento, y entre sus funcionarios se prevé el nombramiento de un ingeniero y un arquitecto municipal, cargo éste ocupado inicialmente, por Mauricio Jalvo Millán, interesante personaje que había ejercido en Madrid, muy comprometido políticamente2.

En el plano económico Melilla pasó a ser un activo centro comercial y financiero, como capital de la zona norte del Rif oriental, y único puerto de importancia en todo el litoral, con las ventajas que le proporcionaba el ser puerto franco desde 1863 y entrada comercial al norte de Marruecos ${ }^{3}$.

El cambio fue tan radical que la población se multiplicó, y si los datos del plano de Villalba de 1800 , señalan poco más de 2.000 habitantes y en el último decenio del siglo 3.000, en 1909 se censaron 16.754. Fueron los acontecimientos bélicos los que aceleraron el proceso poblacional y urbano porque como consecuencia de las cam-

1 SARO GANDARILLAS, F.: "Municipalidad y administración local, antecedentes a la constitución de Ayuntamiento de Melilla", Aldaba $\mathrm{n}^{\circ}$ 3, Melilla, UNED, 1984, págs. 27-40.

2 BRAVO NIETO, A.: La ciudad de Melilla y sus autores. Diccionario biográfico de arquitectos e ingenieros, finales del siglo XIX y primera mitad del XX. Melilla, Ciudad Autónoma, 1997, págs. 45-52.

3 CAMACHO MARTÍNEZ. R.: "El proyecto de puerto de Melilla de 1891, eslabón de la política africanista española", en Melilla en la historia, sus fortificaciones, Seminario celebrado en Melilla en mayo de 1988 Madrid, ICRBC, 1991, págs. 43-57. 
pañas militares que España emprende en Marruecos, en ese año un ejército de 22.000 hombres se estableció en la ciudad que experimentó un crecimiento desmesurado por el llano y lomas circundantes, y, contando a la población civil que las operaciones militares arrastran, alcanzó la cifra de 41.000 habitantes $^{4}$. La ciudad presidio se transformó rápidamente, conjugándose la sobriedad castrense con la exuberancia ornamental de los nuevos estilos burgueses.

Pero antes de construir se planificó y urbanizó, trabajo que acometieron los ingenieros militares. La urbanización de la moderna ciudad de Melilla tiene unas peculiaridades específicas, ya que no se trató en su totalidad de un ensanche, aunque por extensión y en relación con la ciudad vieja así se le llame, sino que se puede hablar de una ciudad construida "ex novo", porque la configuración de ese nuevo espacio urbano se concentra en un periodo reducido de tiempo que se delimita entre 1880 y 1935, y además, porque al ser plaza militar, son las necesidades defensivas las que condicionan su urbanismo, y su rápido desarrollo por el hecho de que una vez concedido el Protectorado Español en Marruecos, Melilla se convierte en un centro estratégico de primer orden. ${ }^{5}$

En un periodo inicial la urbanización, que se desarrolla entre 1859 (fecha del primer tratado para la ampliación de límites) y 1893 (guerra de Margallo) se centra en un ensanche intramuros y otro fuera de éstos pero arropado por la fortificación. Entre 1893 y 1921 (Desastre de Annual) se lleva a cabo otra importante fase de urbanización, a partir del cuarto recinto, en el llano ganado al rio de Oro, cuyo curso se había desviado en la etapa anterior; el Plan del ingeniero militar Eusebio Redondo (1906), muestra una disposición en retícula ortogonal enmarcada por ejes viarios de primer orden, con manzanas generalmente achaflanadas, ocupando su centralidad el Parque Hernández y limitado por barrios extremos. El Plan de Urbanización y Ensanche del también ingeniero José de la Gándara (1910) que supone la expansión de la ciudad hacia los límites y la conexión de todos los barrios, plantea también una importante actuación en la zona que había quedado entre las murallas (transformando el barrio de El Mantelete) y el ensanche anterior, una gran plaza circular de la que parten avenidas radiales. Es justo en esta fase donde tenemos que situarnos, aunque la evolución de la ciudad continúa en un cuarto periodo, entre 1921 y la guerra civil, que por no haberse aplicado la planificación urbana y la afluencia masiva de inmigrantes puede considerarse un caos urbano. Un último periodo que arranca posguerra apenas afectó al espacio urbano, y con la finalización del Protectorado, en

4 SARO GANDARILLAS, F.: Estudios melillenses. Notas sobre urbanismo, historia y sociedad en Melilla. Ayuntamiento de Melilla, 1996 págs. 72 y ss.

5 ARGENTE DEL CASTILLO, F. J.: “El urbanismo en la Melilla contemporánea”, en CAMACHO MARTÍNEZ R. (Coord.) Expediente para la declaración de Melilla "Ciudad Patrimonio de la Humanidad" (texto inédito). 
1956, el desarrollo inmobiliario prácticamente desaparece6.

En los solares que rodean esa gran plaza redonda, la que sería la Plaza de España y, en general en esa Melilla en crecimiento, surgió una arquitectura de formas atrayentes, cuyo rasgo más característico fue la ornamentación, fachadista fundamentalmente. Es pues un concepto de máscara en el que se mezclan la riqueza, ostentación y apariencia, porque los arquitectos e ingenieros se dieron cuenta de que se podía acceder a una cierta novedad variando el lenguaje formal, realizándose una lectura del movimiento como fórmula de modernidad y medio de renovación, como indica Antonio Bravo, y sólo cuando empiece a despojarse de esta máscara las estructuras determinarán un lenguaje más sincero, verdaderamente moderno, aunque no sin establecer pasarelas intermedias. Y esta arquitectura ornamentada, que hemos definido por extensión como modernista, aunque inicialmente provocó el rechazo de figuras tan importantes como Torres Balbás ${ }^{7}$, ofrece uno de los panoramas más atrayentes de la ciudad y la va configurando en una etapa más amplia de lo que habitualmente se señala para el Modernismo; porque con este término se abarca una realidad estilística que incluye las derivaciones clasicistas, las corrientes historicistas, el eclecticismo, el modernismo en sus diferentes vertientes, y el art déco zigzagueante y aerodinámico con los que entra el movimiento moderno ${ }^{8}$.

Melilla ha sido reconocida para la historia de la arquitectura contemporánea a partir de la exposición del Modernismo, celebrada en 19699. Sin embargo, como hemos visto, la evolución de esta arquitectura es algo más amplia, pero hablar de Modernismo en Melilla es hablar del arquitecto catalán Enrique Nieto, colaborador de Gaudí y formado en la Escuela de Arquitectura de Barcelona, entre otros por Domenech y Montaner. Nieto se estableció en Melilla en 1909, y su actividad, que llega hasta 1940 y acusa los cambios estilísticos, fue determinante en el cambio de imagen de la ciudad ${ }^{10}$. A Nieto le llovieron los encargos por parte de una burguesía

6 ARgente DEL CASTILLO, F. J.: "Evolución urbana de Melilla", en BRAVO NIETO, A. y FERNÁNDEZ URIEL, P. Historia de Melila. Ciudad Autónoma de Melilla, 2005, págs. 740-762.

7 TORRES BALBÁS, L.: “La arquitectura española en Marruecos", en Arquitectura, vol V, 1023 Págs. 139142. El autor considera que en la zona del protectorado español de Marruecos se ha construido deprisa y mal y con absoluto desconocimiento del país, y lamenta que no se hubiera llevado a cabo una arquitectura resultante de la depuración de lo aprendido en la Espana del sur, conservando carácter y estilo de sus ciudades y edificando junto a éstas barrios modernos que no desentonaran con su estilo. Considerando que se ha construido una Melilla "moderna de calles rectas, manzanas regulares, casas horribles de cemento, de un estilo bárbaro, lleno de adornos pegados", cercanos a un espantoso pseudomodernismo.

8 BRAVO NIETO, A.: La construcción de una ciudad europea en el contexto norteafricano. Arquitectos e ingenieros en la Melilla contemporánea. Ciudad Autónoma de Melilla y Universidad de Málaga, 1996, págs 441 y ss.

9 BASSEGODA NONELL, J. (et al.): El Modernismo en España. Catálogo de la exposición celebrada en el Casón del Buen Retiro de octubre a diciembre de 1969. Madrid, Dirección General de Bellas Artes, págs. 10, 38-39 у 178

10 GALLEGO ARANDA, S.: Enrique Nieto en Melilla: La ciudad proyectada. Centro Asociado de la U.N.E.D. en Melilla y Universidad de Granada, 1996. 
que, como todas las europeas sintoniza con las nuevas actitudes estético-filosóficas, reivindicando lo subjetivo y la libre fantasía, que ha ascendido merced a su posición económica y quiere ofrecer una imagen de la ciudad diferente de la del periodo más marcado por la estructura militar y se identifica con estas formalizaciones novedosas que les deslumbra.

\section{LOS NUEVOS PROYECTOS ARQUITECTÓNICOS.}

A comienzos de, segundo decenio del siglo $X X$ ya habían empezado a surgir importantes viviendas de renta que iban cerrando la Plaza de España. Con proyecto del arquitecto malagueño Manuel Rivera Vera a partir de 1910 se construye, para el también malagueño D. Félix Sáenz, un edificio muy significativo con esquina a la Avenida $n^{\circ} 2$, que responde al particular modernismo geometrizante de Rivera, siguiendo modelos europeos (que en Málaga se relaciona con los edificios de $\mathrm{C} /$ Santa María $n^{\circ} 25$ y C/ Alarcón Luján $n^{\circ} 1^{11}$ ). Y en la esquina opuesta cerraría la Avenida un edificio de Nieto de 1915 que construye para D. David Melul, que con sus torrecillas cupuladas, el ornamentado peinetón curvo del eje central, la elaborada decoración figurativa y de guirnaldas que conviven con los círculos de la Secesión vienesa, muestra la influencia de su maestro Domenech y Montaner.

El solar más destacado de los que rodean la plaza, el que marca la continuación con la zona del puerto, a través del Paseo del General Macías, que lo limita, será objeto de importantes propuestas. En la revista La Construcción Moderna ${ }^{12}$ se publicó en 1922 el proyecto para edificio de la Comandancia General, firmado por el ingeniero militar Mariano Campos Tomás (nacido 1870). En abril de 1923 se seguía trabajando sobre este mismo proyecto, y el ingeniero militar Juan García San Miguel y Uría (nacido 1899) realizaba un tanteo, o anteproyecto para construir este edificio, aunque éste sería el último trabajo que conocemos, olvidándose posteriormente su instalación en este espacio urbano ${ }^{13}$. En este solar, de forma pentagonal y con su lado principal adaptado a la forma curva de la plaza, se distribuyen las dependencias simétricamente a ambos lados del vestíbulo principal y de la escalera

\footnotetext{
11 RODRIGUEZ MARIN, F. J.: "Manuel Rivera Vera (1879-1940).Último eslabón de dos generaciones de arquitectos malagueños" II, en Boletín de Arte $n^{\circ} 13-14$, Universidad de Málaga, 1992-93, pág. 243. GALLEGO ARANDA, S.: "Un proyecto del arquitecto D. Manuel Rivera Vera para D. Félix Sáenz en Melilla: el edificio n 2 de la Avenida", en Boletín de Arte n 15, Universidad de Málaga, 1994, págs. 239-256.

12 "Proyecto de Edificio para Comandancia General de Melilla". La Construcción Moderna, 1922, págs. 16 y 17.

13 BRAVO NIETO, A.: La ciudad de Melilla y sus autores, pág. 53. La construcción... pág. 649. Agradezco al profesor Bravo Nieto que me haya facilitado las imágenes de este proyecto y los del Ayuntamiento. Un original del proyecto, acuarelado, se encuentra en el interior de la actual Comandancia General de Melilla, otra copia del proyecto en un fotograbado de La Construcción Moderna, 1922; Op. Cit. Los planos de García de San Miguel, en el antiguo archivo de la Comandancia de Obras de Melilla. Planos sueltos.
} 


2: artículos Rosario Camacho Martinez
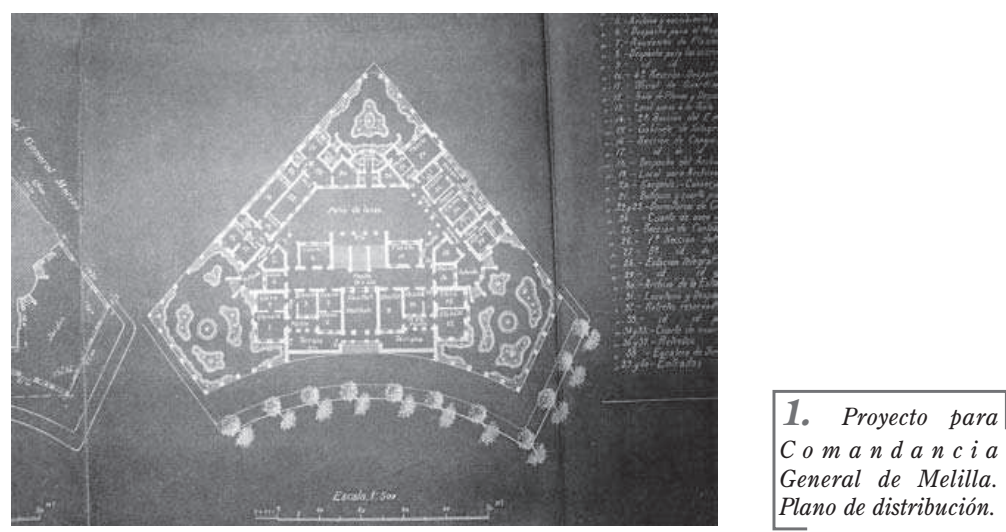

que abre a un patio de luces, centro de las estancias de la zona posterior, dejando en los tres vértices del solar atractivos patios ajardinados. Su alzado exterior responde a un exaltado regionalismo ya que recoge los estilemas de obras renacentistas como el Palacio de Monterrey de Salamanca, con el que trata de coincidir a través de elementos tan significativos como sus torres cuadradas rematadas por tracería y pináculos [1-2]

El alzado exterior nos ofrece un edificio clásico y monumentalista con un piso inferior más sólido, con escasos huecos y mimetizando sillería, mientras que en el piso noble, manteniendo los ejes de huecos se abre un amplio fenestraje entre columnas coronado por frontoncillos; en esta fachada principal se destacan las dos torres cuadradas de los extremos, con galerías en el piso superior y el remate de cresterías, y el cuerpo central que se adelanta formando un airoso pórtico de acceso sobre el que apoya el balcón central, coronándose por encima de la balaustrada con un cuerpo triple rematado por frontón al estilo de otra obra clásica: la universidad de Alcalá. Las fachadas laterales se abren definiendo el espacio pentagonal que marca la transición con las torres unos tramos curvos al que acceden las escaleras laterales, coronándose en el cuerpo superior por amplias cristaleras.

Aunque la moderna Melilla se definía más por el cosmopolitismo y no proliferó mucho en ella el regionalismo, un proyecto para la sede de la más importante representación militar tenía que ser bien significativo ${ }^{14}$. Así que no es extraño que se eligieran estas formalizaciones que, desde comienzos del siglo XX se había convertido en el estilo nacional por excelencia. Su uso como revival es anterior, ya que en

14 BRAVO NIETO , A.: La construcción..., pág. 649. 


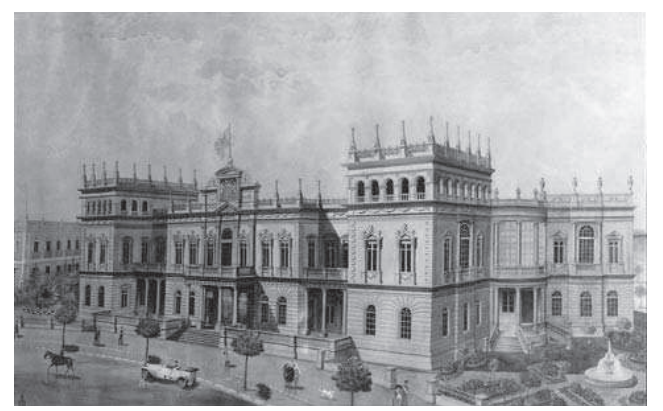

2. MARIANO CAMPOS TOMÁs, Proyecto para Comandancia General de Melilla.

la Exposición Universal de París, de 1867, el Pabellón Español que construyera el arquitecto Jerónimo de la Gándara, es más que una evocación del Palacio de Monterrey que construyera Rodrigo Gil de Hontañón en 1539. Es cierto que no se volvió a hacer uso de este revival "neoplateresco" en las exposiciones del XIX, tal vez porque está exento del pintoresquismo que requerían estos pabellones, aunque también se ha apuntado que su rechazo pudo estar determinado porque fue elegido por un gobierno reaccionario, sólo un año antes de la revolución liberal que derrocó a Isabel II. Sin embargo este neoestilo irá afianzándose, ya en la exposición de París de 1900 el pabellón construido por Urioste responde al neorrenacimiento, para aparecer en los primeros años del siglo completamente legitimado como estilo nacional 15. Por otro lado el ingeniero militar José de la Gándara, tras su destino en Melilla, proyectó la Academia de Caballería de Valladolid, también en "estilo Monterrey". Y volviendo a Melilla su huella podemos apreciarla en la Residencia de Oficiales (C/ General Astilleros), de 1944, que con una fábrica mucho más austera y racionalista, no prescinde de las torres laterales incluso con la crestería ${ }^{16}$. Y al reformarse la Comandancia General, (C/ Luis de Sotomayor), en 1948, se diseñó su portada principal con un frente de columnas que sirven de soporte al balcón de apariciones, que parece trasladada de este proyecto ${ }^{17}$.

Pero el solar no albergaría un edificio militar sino que en él se levantará el edificio civil más representativo de la ciudad, el Ayuntamiento, hoy Palacio de la Asamblea.

15 BUENO FIDEL, Ma J.: Arquitectura y Nacionalismo (Pabellones españoles en las exposiciones universales del siglo XIX). Colección 2 A, Colegio de Arquitectos y Universidad de Málaga, 1987, págs. 94-99. NAVASCUÉS PALACIO, P.: Arquitectura española 1898-1914. Col. Summa Artis vol. XXXV. Madrid, Espasa-Calpe, 1993, págs.668 y ss.

16 BRAVO NIETO, A.: La construcción..., págs. 650-651.

17 BRAVO NIETO, A.: La ciudad de Melilla y sus autores, pág. 52 y 53. 
Desde 1926 se había iniciado una etapa de colonización en la que Melilla desempeña un papel muy activo. Es la época cumbre de su expansión económica que se manifiesta nuevamente en el aumento de población y en el volumen de la construcción en la que resalta su monumentalismo y el tránsito hacia otras formas más racionalistas que se asumen a través del art déco, el estilo de los decorativos años 20 , entendido éste como una corriente tendente hacia un ideal de modernidad formal, a través de la cual se intenta despegar del Modernismo, siendo el edificio de mayor calidad, el Monumental Cinema Sport, una de las obras más logradas del déco cosmopolita, proyectado por el arquitecto de Cartagena, Lorenzo Ros Costa, en 193018. Esta obra tuvo un peso importante que pudo suponer el inicio del déco en Melilla, pero ya llegaba también por otros caminos: además de la cultura arquitectónica, la obra de Jalvo y Edo y la del arquitecto Francisco Hernanz, máximo responsable de las tendencias más racionalistas 19

En esta transición hacia el racionalismo, y con una fuerte presencia del art déco, hay que citar los proyectos de los que surgirá el edificio del Ayuntamiento.

Una vez constituida la Junta Municipal y dado el paso a Ayuntamiento, en noviembre de 1929 se convocó concurso para construir un palacio municipal, para el que dictó las bases el arquitecto municipal Mauricio Jalvo 20.

La convocatoria del concurso interesó a numerosos estudios y arquitectos ${ }^{21}$, solicitando la prórroga del plazo de presentación, que expiraba finales de abril, pero no se admitió. Se presentó un anteproyecto, de Tomás Mur y cinco proyectos, que firmaban Luis López y López (Madrid). Javier Barroso Sánchez-Guerra con Felipe López Delgado (Madrid). Manuel Cárdenas, Gonzalo Cárdenas e Ignacio Zulueta (Madrid). Joaquín Ma Fernández Cabello y Luis Ferrero (Madrid). Manuel Muñoz Monasterio y Mariano Rodríguez Orgaz (Madrid).

Revisado el material enviado, se declaró fuera de concurso el anteproyecto de Mur y se rechazó el de Cárdenas y Zulueta. El interventor informó era el más adecuado para su realización, con gran diferencia sobre los demás, el proyecto de Luis

18 CAMACHO MARTíNEZ, R.: "El arquitecto Lorenzo Ros Costa y la difusión del art déco en Melilla", Seminario Arquitectura y Ciudad celebrado en 1989. Madrid, Ministerio de Cultura, 1992, págs. 55-66.

19 BRAVO NIETO, A.: La construcción...págs. 581 y ss.

20 Archivo Municipal de Melilla (A.M.ME). Sección de Obras y proyectos. Leg. 29, 1. Estos proyectos han sido estudiados también por los profesores Bravo Nieto: La construcción...págs. 463 y 578 y Gallego Aranda: Enrique Nieto...págs. 260-264.

21 A.M.ML. Leg. 29, 1. De enero de 1930 hay una relación de arquitectos que solicitaron información para participar: Oficina Moderna de Arquitectura de Málaga (firma Ricardo Santacruz); Juan Bautista Carles, de Valencia; Tomás Muñoz Lapeyrade, de Sevilla; Ricardo Pérez, arquitecto municipal de Salamanca; Ulargui Moreno, de Madrid; Luis Ferrero y Llusía, de Madrid; Francisco Massot, de Madrid; Manuel Cabaynes Mata y Antonio Callejo Álvarez, de Madrid, Antonio Delgado Roig, de Sevilla; Andrés Ceballos, de Madrid. Además Juan Jaúregui Briales, Secretario de la Asociación de Arquitectos de Málaga, solicita que se prorrogue el plazo porque varios arquitectos de Málaga quieren participar. 
Ferrero y Joaquín $M^{a}$ Fernández Cabello, por responder al estilo neoárabe, quizá porque así se remitía a aquella etapa de nuestro pasado de convivencia pacífica entre culturas, y esos matices ideológicos interesaban en Melilla. No obstante se declaró desierto el primer premio de 12.000 ptas., concediéndose tres segundos de 6.000 ptas: a éstos, al proyecto de Luis López y al de Barroso y López Delgado, así como un accesit al proyecto presentado por Manuel Muñoz Monasterio y Mariano Rodríguez Orgaz.

Pero hasta enero de 1932, siendo ya Enrique Nieto arquitecto municipal, no se pensó en acometer la obra, y como había quedado claro que el proyecto más apropiado para Melilla era el que presentaron Ferrero y Fernández Cabello, les propuso la comisión que fuesen a Melilla para modificar algunos aspectos. En abril se recibió el proyecto reformado así como los honorarios y gastos, que parecieron excesivos al Ayuntamiento. El conflicto estaba planteado porque enterados los arquitectos autores del proyecto que el Ayuntamiento había encargado a Nieto reformarlo y adaptarlo, en noviembre de 1932 se quejan de la situación y acusan al arquitecto de infringir la ley de Propiedad Intelectual así como los Estatutos de los Colegios Oficiales de Arquitectos (aprobados en junio de 1931) y comunican esta actuación a la Junta de Depuración Personal de Colegios de Arquitectos, insistiendo en que su proyecto no se puede llevar a la práctica ni dirigirlo otro arquitecto mientras no se les abonen los honorarios. Pero la condición 11 del concurso indicaba que los proyectos ganadores quedarían de propiedad de la Junta Municipal, que podría encargar la dirección y ejecución a quien quisiera. Por tanto Ferrero y Fernández Cabello habían perdido la propiedad de su proyecto 22 . Para terminar este conflicto y empezar la obra, que contribuiría a paliar la crisis de paro obrero de Melilla, la comisión de Fomento recomendó que si no era posible reformar el proyecto, fuese anulado haciéndose otro de acuerdo con las posibilidades económicas de aquel momento, lo que realizaría Nieto quien, evidentemente, tuvo en cuenta los proyectos presentados y muy especialmente el del conflicto, aunque él mismo había indicado que le parecía más apropiado para el Protectorado que para Melilla23.

Analizando los proyectos ${ }^{24}$ podemos comprobar que van de un mayor eclecticismo a formas cuyo lenguaje implica una intencionalidad expresiva y parlante.

22 A.M.ML. Leg. 29, 1. Todavía en 1934 insistían en su protesta al comunicar al Colegio de Arquitectos de Madrid que el Ayuntamiento no podía disponer de los proyectos ya que la Junta había roto las bases cuando cambió los premios otorgando tres segundos y no el primero; la Junta de Fomento decidió que se le abonasen los honorarios deduciendo 6.000 ptas del premio, porque reclamaban las 12.000 del primero. 23 BRAVO NIETO, A: La construcción, pág. 463.

24 Las fotografías de los cuatro proyectos principales fueron publicadas en Junta Municipal de Melilla, Memoria de su actuación 1927-1930. Melilla: s/f (pero 1931), entre las páginas 74 y 75 . Con un carácter divulgativo, véase el trabajo de CARMONA PACHÓN, F.: "Proyectos frustrados y algunos posteriormente realizados". La Voz, 13 de febrero de 1994; pág. 10 y 11. 


2: artículos Rosario Camacho Martinez

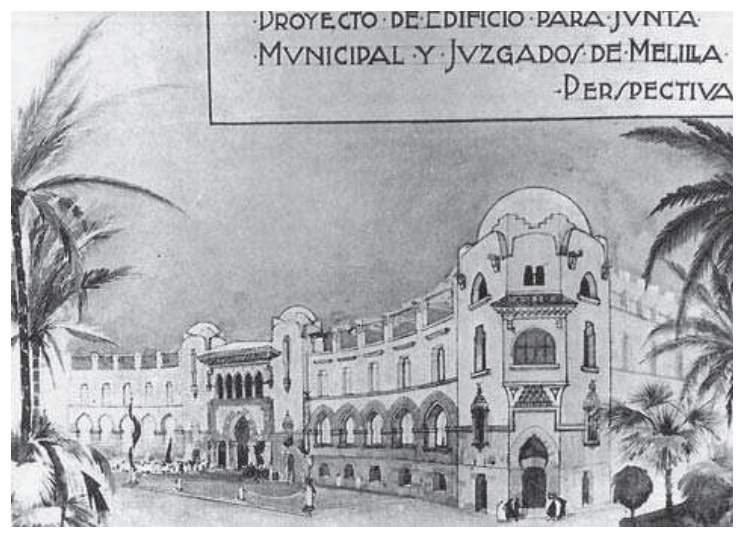

3. LUIS FERRERO $Y$ JOSÉ $M^{a}$ FERNÁNDEZ CABELLO, Proyecto para Ayuntamiento de Melilla.

En este sentido destaca el proyecto elegido, el de Luis Ferrero y José $\mathrm{M}^{\mathrm{a}}$ Fernández Cabello, que era el más próximo a la estética neoárabe. Al considerarlo la Junta Municipal como el que "reune mejores condiciones con gran diferencia sobre los demás", apostaba por una propuesta con una carga ideológica fuerte, porque la utilización de esas formas en el contexto norteafricano no se entendía como un exotismo sino una reexportación de formas, recordando en Marruecos aquella arquitectura "excelente" que allí se había olvidado y que sí se había conservado en España, tesis que vienen a coincidir con las del escritor africanista Basilio Paraíso, y fueron muchas las obras realizadas con esta estética en la zona del Protectorado, incluso en el ámbito castrense de Melilla25.

El diseño presenta su curvada fachada entre dos torres rematadas por cúpula, con galería de arcos de herradura apuntados y otros quebrados, más próximos al art déco. La portada principal, con galería y tejaroz, también enmarcada entre dos torrecillas, conjuga el diseño del arco triunfal con ecos de las grandes obras de la arquitectura mudéjar, como el Alcázar de Sevilla. Porque las citas están muy cuidadas, se separan de otras obras del estilo "neoárabe arqueológico" realizado en la ciudad, adaptándose inteligentemente al art déco que se vislumbra detrás de este diseño [3].

En los otros tres proyectos cambia la composición de la fachada, destacando un hito vertical sobre el eje de la portada principal.

El diseño más clásico fue el presentado por Luís López y López, que en la memoria justifica este estilo remontándose incluso a los "condes de provincia" en

25 BRAVO NIETO, A.: La construcción..., pág. 449 


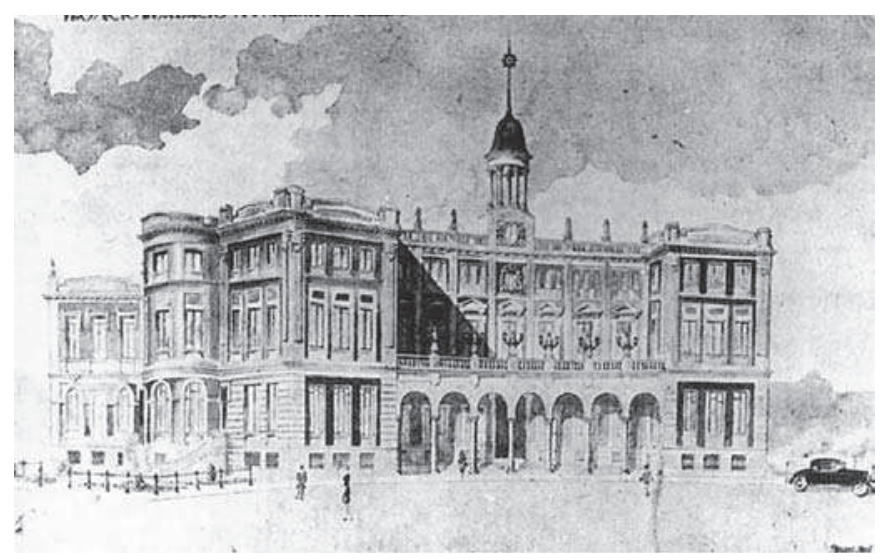

4. LUIS LÓPEZ Y LÓPEZ. , Proyecto para Ayuntamiento de Melilla.

Castilla y reconoce su inspiración en "los clásicos de la arquitectura civil española", adaptándolo a las necesidades de este edificio, que sigue diferentes modelos ${ }^{26}$. El resultado es un conjunto ecléctico y monumentalista que en su fachada principal, con pórticos y cuerpo noble con balconada, encajada entre dos torres cúbicas, remite a formas que van desde el Palacio Real de Madrid al Ayuntamiento de Cádiz, y con el remate de la torre central se hermana con otras tipologías de ayuntamientos de finales del XIX. Pero trata de aligerar el bloque cúbico de la fachada disponiendo los accesos laterales, que conducen a los diferentes juzgados, mediante rotondas $y$ escaleras que se adaptan a la forma curva de éstas [4].

Describe el interior con un patio cubierto con claraboya que abre a dos plantas y de allí arranca la escalera de honor, con una gran vidriera al fondo, dejando uno de los espacios de la rotonda para sede del museo municipal. La relación de los pormenores decorativos: frisos de madera, molduras de escayola imitando artesanías renacentistas, dejan patente la intención clasicista y representativa de esta propuesta.

El proyecto de Manuel Muñoz Monasterio y Mariano Rodríguez Orgaz presenta una composición equilibrada resaltando su sencillez y sentido clásico en el trazado de los huecos; también destaca el hito vertical de su torre central, que integra

26 A.M.ML. Leg. 29, 3. 


Q articulos Rosario Camacho Martínez

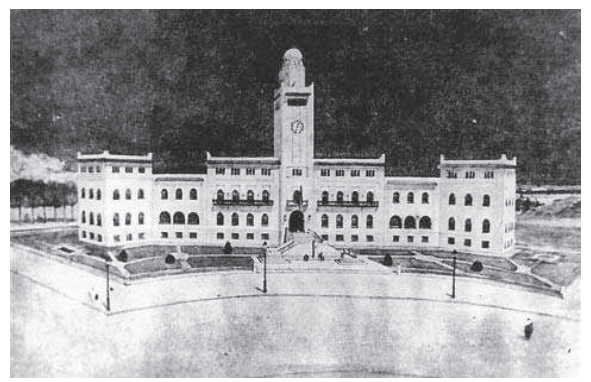

5. MANUEL MUÑOZ MONASTERIO Y MARIANO RODRÍGUEZ ORGAZ, Proyecto para Ayuntamiento de Melilla.

el acceso principal, de clara inspiración en obras de la Secesión vienesa [5].

El proyecto presentado por Javier Barroso y Felipe López Delgado es el más interesante 27 . Como ellos mismos señalan en la memoria, quieren enlazar con la estética neoárabe, pero conociendo la capacidad de la ciudad para comprender el abuso del neoárabe y neomudéjar, ofrecen una "interpretación colonial", convencidos del sentido moderno de su propuesta, en la que al dominar la funcionalidad y sencillez, se aproxima mucho más al verdadero sentido del estilo árabe.

Realmente es un diseño notable que entronca asimismo con el art déco, definiendo el edificio por la ponderación de las masas, cúbicas y racionalistas, en cuya superficie contrasta el uso del ladrillo sobre revoco blanco, restringiéndose los huecos. En la fachada resalta la torre central, prisma vertical de notable belleza, que los autores no consideran un capricho decorativo obligado por la tradición del tema de los ayuntamientos, sino que es totalmente utilitaria al permitir alojar los depósitos que, en altura proyectan el agua a presión. Y llama la atención por su sobriedad decorativa.

\section{LA MATERIALIZACIÓN DEL EDIFICIO.}

Aunque Enrique Nieto está trabajando sobre este proyecto desde 1932, la ejecución de la obra no se acometería hasta después de la guerra, inaugurándose en 194928. El esquema compositivo arranca del proyecto de Ferrero y Fernández Cabello manteniendo las macizas torres que cierran la curva fachada y el bloque de la portada principal entre dos torrecillas más esbeltas y donde se acumulan las evocaciones historicistas. Pero, inteligentemente, el diseño, que combina regularidad y monumentalismo con un sentido de la proporción verdaderamente clásico, se ha

27 A.M.ML. Leg. 29, 2.

28 CAMACHO MARTINEZ, R.: "Las sugestiones del art déco en la arquitectura de Melilla", en Boletín de Arte nº 7 , Málaga 1986, pág. 157. 


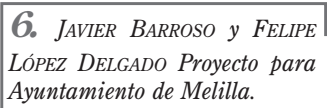

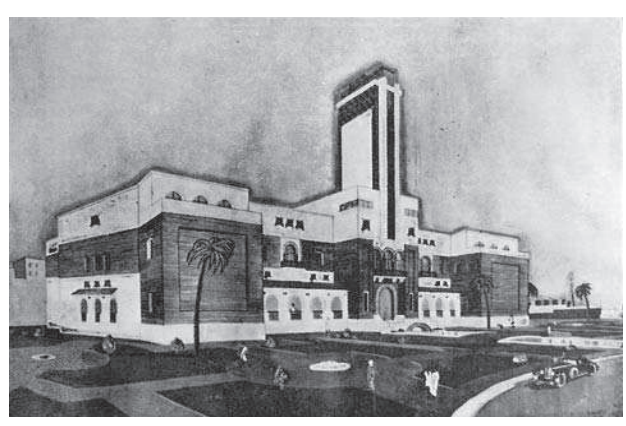

transformado en art déco, como se aprecia en los huecos de dintel quebrado, los placados con estlizaciones florales, las celosías, la tipología de los balaustres y elementos de soporte del pórtico, poliédricos en su tercio superior, el extraño capitel más chipriota que jónico del piso principal o el diseño de la reja con líneas de abanico de vértices opuestos. También aquí podemos encontrar otras fuentes, más afines a la Secesión vienesa, como el palacio Stoclet de Bruselas, obra de Hoffman de 1905, con el que pueden relacionarse las torrecillas centrales, también evocadas en la torre central del proyecto de Muñoz Monasterio y Rodríguez Orgaz, lo que demuestra que beben en fuentes comunes [7].

En el interior hay detalles muy sugestivos, que corresponden al proyecto de los años treinta, desde el zaguán con pilares de mármol negro de capiteles ménsula, el hall donde destaca la rotundidad de sus pilares poligonales de mármol rojo y negro, los marcos de puertas con ricos placados de mármol, la doble escalera imperial, o la balaustrada de evocación egipcia, aunque ésta es diseño de $1948^{29}$. Entre los salones destaca el de Plenos, donde con su división del espacio mediante diafragmas de perfil mixtilíneo, las esbeltas pilastras decorativas coronadas por palmetas y volutas, el enrejado de las sobrepuertas que recuerda las rejas de A. Piquet en la exposición de París de $1925^{30}$, y el estucado crema enriquecido por toques dorados, se recrea un ambiente elegante y sofisticado, acorde con la línea del estilo. Pero esos detalles resultan tímidos y quedan ofuscados al conjugarse con formas más tradicionales y barroquizantes, obra ya de los años cuarenta, así como el mobiliario que se seleccionó para vestir el edificio e imprimirle su carácter institucional, ofreciendo un fuerte contraste con la modernidad del exterior.

29 GALLEGO ARANDA, S.: Enrique Nieto (1880-1954). Biografía de un arquitecto. Melilla. Fundación Melilla Monumental, 2005, pág. 174

30 BATTERSBY, M.: The decorative twenties. Studio Vista, U.S.A., 1976, pág. 25. 


\section{Q: articulos Rosario Camacho Martínez}

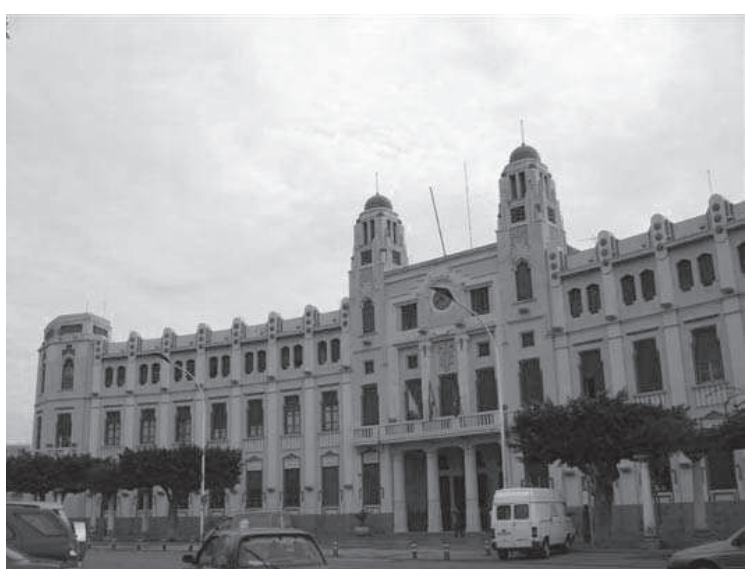

7. ENRIQUE NIETO Y NIETO, Ayuntamiento de Melilla.

El edificio del Ayuntamiento de Melilla es un bien protegido. Por un lado por encontrarse en el centro de Melilla, catalogado como Bien de Interés Cultural por RD 2753/1986 de 5 de diciembre, y por otro, como pieza aislada, cuenta con la máxima protección en el Catálogo de arquitectura de Melilla. 1895-196131.

Además, desde 2004 se está realizando un plan especial de protección del ensanche modernista de Melilla, dirigido por el arquitecto D. Juan Armindo Hernández Montero y por el historiador del Arte D. Antonio Bravo Nieto, que está en vías de aprobación, y donde el edificio obtiene un alto grado de protección, tanto en su exterior como en sus espacios interiores.

31 BRAVO NIETO, A. e HINOJO SÁNCHEZ, D.: Catálogo de arquitectura de Melilla. 1895-1961. Melilla: Consejería de Fomento, 2003. 